\title{
Characterization of the Dual Function of ATXR5 PIP motif in PCNA and Nucleosome Binding
}

\author{
Hossein Davarinejad, Monika Joshi, Narimane Ait Hamou, Jean-François Couture \\ Ottawa Institute of Systems Biology, Department of Biochemistry, Microbiology, and Immunology, \\ University of Ottawa, Ottawa, ON, Canada K1H 8L1
}

ATXR5 (Arabidopsis trithorax-related protein 5) is a SET domain lysine methyltransferase (KMTase) playing an important role in genomic stability and DNA replication (Jacob et al. 2009). Previous studies established that the plant homeodomain (PHD), the proliferating cell nuclear antigen (PCNA) interacting peptide (PIP) and the SET domain of ATXR5 contribute to its biological roles (Bergamin et al. 2017). While our previous findings established that ATXR5 SET domain preferentially methylate histone H3.1 on lysine 27 (H3K27) when incorporated in a nucleosome core particle (NCP) (Jacob et al. 2014) and that its PHD domain is important for ATXR5 activity (Bergamin et al. 2017), the role of its PIP motif has remained poorly explored. In this study, we show that two molecules of ATXR5 binds to a PCNA trimer and the crystal structure of PCNA bound to ATXR5 PIP demonstrates that a di-phenylalanine motif is important for high affinity binding of the methyltransferase to PCNA. Surprisingly, increasing concentration of PCNA blocks ATXR5 KMTase activity on the NCP suggesting that the NCP and PCNA have overlapping binding sites on ATXR5. Accordingly, ATXR5 PIP mutants show lower methyltransferase activity demonstrating that this region of the enzyme is important for high affinity binding to the NCP. Our studies collectively demonstrate that the PIP motif of ATXR5 interacts with PCNA and performs a secondary function by regulating the interaction of the enzyme with the NCP.

\section{$\underline{\text { References }}$}

Bergamin E, Sarvan S, Malette J, Eram MS, Yeung S, Mongeon V, Joshi M, Brunzelle JS, Michaels SD, Blais A, et al. 2017. Molecular basis for the methylation specificity of ATXR5 for histone H3. Nucleic Acids Research. 45(11): 6375-6387.

Jacob Y, Bergamin E, Donoghue MTA, Mongeon V, LeBlanc C, Voigt P, Underwood CJ, Brunzelle JS, Michaels SD, Reinberg D, et al. 2014. Selective Methylation of Histone H3 Variant H3.1 Regulates Heterochromatin Replication. Science. 343(6176): 1249-1253.

Jacob Y, Feng S, LeBlanc CA, Bernatavichute YV, Stroud H, Cokus S, Johnson LM, Pellegrini M, Jacobsen SE, Michaels SD. 2009. ATXR5 and ATXR6 are H3K27 monomethyltransferases required for chromatin structure and gene silencing. Nature Structural \& Molecular Biology. 16: 763. 